\title{
ESTUDO DA ESTABILIDADE TÉRMICA DE FIBRA QUERATINA - KERATIN FIBRE (KF) - DE PENAS DE FRANGO PARA OBTENÇÃO DE CARBONO - CARBONISED FEATHERS (CF)
}

\author{
D. D. Belarmino', R. Ladchumananandasivam ${ }^{1}$, L. D. Belarmino ${ }^{2}$, S. M. B. Andrade ${ }^{1}$, A. O. Galvão ${ }^{1}$ e L. M. \\ Ribeiro ${ }^{1}$. \\ 'Programa de Pós Graduação em Engenharia Mecânica, Laboratório de Engenharia Têxtil \\ 2 Programa de Pós Graduação em Química, Laboratório de Meio Ambiente e Analítica - UFRN \\ debelarmino@yahoo.com.br - rasiah@ufrnet.br
}

Artigo submetido em abril/2012 e aceito em julho/2012

\section{RESUMO}

As KF de penas de frango foram submetidas à análise térmica - Termogravimétrica (TG) em sua forma natural e carbonizada. A partir das curvas obtidas por TG e sua Derivada (DTG) da pena natural (KF), tratada com Hidróxido de Sódio (KFNaOH), semicarbonizadas - "Semi Carbonized Feathers" "Clear" (SCFC) e "Dark" (SCFD), buscou-se um melhor ciclo de carbonização (pirólise) para esse tipo de precursor. O trabalho também trata como um dos focos, o estudo comparativo do comportamento térmico desses materiais. Uma parte da KF foi tratada com hidróxido de sódio $(\mathrm{NaOH})$, e observou-se pelo o resultado TG/DTG que a resistência térmica do material não foi influenciada. Outra parte de $\mathrm{KF}$ foi semicarbonizada $\left(220^{\circ} \mathrm{C}\right)$ sem controle do ambiente térmico e taxas de aquecimento e resfriamento, apresentando colorações diferentes, mais clara (SCFC) e mais escura (SCFD). Foi constatado nas curvas de TG/DTG, que esse tratamento de carbonização não é ideal para a obtenção de carbono. Também foi verificado nas curvas de TG/DTG que na CF houve um aumento da estabilidade térmica comparada a todas as outras amostras.

PALAVRAS-CHAVE: penas de frango, queratina, análise térmica, pirólise, carbono.

\section{STUDY OF THERMAL STABILITY OF KERATIN FIBRE (KF) OF CHICKEN FEATHERS FOR OBTAINING OF CARBON - CARBONISED FEATHERS (CF)}

\section{ABSTRACT}

Keratin fibers (KF) from chicken feathers were subjected to thermal analysis - Thermogravimetric (TG). This work is based on the curves obtained by TG and its Derivative (DTG) from the natural feather (KF), treated with Sodium Hydroxide (KFNaOH), Semi Carbonized Feathers but Clear (SCFC) and but Dark (SCFD), aimed at achieving a better cycle carbonization (pyrolysis) for this type of precursor. Also the present work compare thermal behavior their. One part of the $\mathrm{KF}$ was treated with Sodium Hydroxide $(\mathrm{NaOH})$, and it was observed from TG/DTG result, that the thermal resistance of the material was not affected. The other part of KF was semi-carbonized $\left(220^{\circ} \mathrm{C}\right)$ without controlled the of environment and the of rates of heating and cooling, thus presenting different colors, of which SCFC was clearer and SCFC was darker. It was noted that from the curves of TG/DTG, this cycle of treatment is not ideal for the obtention of carbon. It was also confirmed in the curves of TG/DTG that feathers carbonized by pyrolysis (CF), there was an increase in thermal stability compared to all other samples.

KEY-WORDS: chicken feathers, keratin, thermal analysis, pyrolysis, carbon. 


\section{ESTUDO DA ESTABILIDADE TÉRMICA DE FIBRA QUERATINA - KERATIN FIBRE (KF) - DE PENAS DE FRANGO PARA OBTENÇÃO DE CARBONO - CARBONISED FEATHERS (CF)}

\section{INTRODUÇÃO}

O aumento da população mundial, associada ao crescimento da poluição ambiental, tem intensificado a procura por novos materiais que venham substituir os derivados do petróleo. As novas tecnologias a serem desenvolvidas devem ser capazes de atender às necessidades do presente sem comprometer o futuro, possibilitando um menor impacto ambiental.

De acordo com o Instituto Brasileiro de Geografia e Estatística (IBGE, 2012a) em 2011 foram abatidos 5,3 bilhões de frangos, indicando um aumento de 5,6\% em relação a 2010. A série histórica da evolução do abate de frangos nos últimos anos pode ser visualizada na Figura 1.

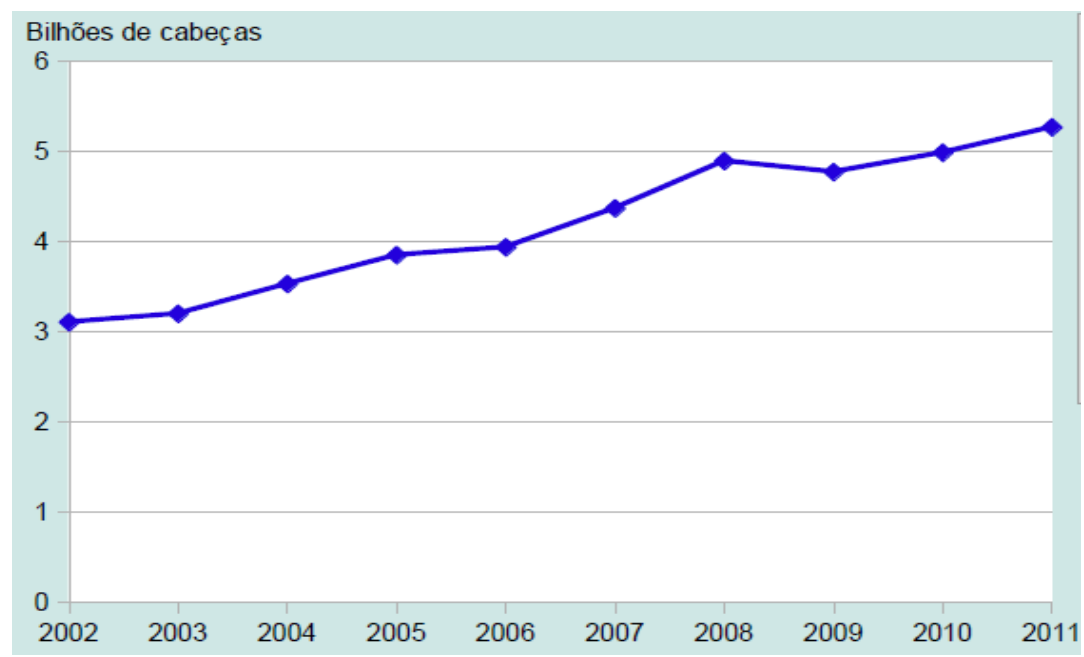

Figura 1 - Evolução do abate de frangos no Brasil nos últimos anos. Fonte: (IBGE, 2012b)

As penas representam 5 a 7\% da massa dos frangos (Moore et al., 2005). Em 2011, Brasil tornou-se o maior exportador mundial dessas aves (AVEWORLD, 2012). Pode-se então, avaliar a disponibilidade em abundância e o desperdício dessa matéria prima. Sabendo que a supressão ou eliminação de resíduos é difícil, onde são queimados, enterrados ou uma pequena parte, reciclado para ração animal. Estes métodos são caros e ecologicamente incorretos, sendo necessária a utilização adequada dessas penas para desenvolvimento de novos processos e produtos, como é o objetivo desse trabalho.

As penas de frangos são fibras naturais proteicas, de origem animal. Essas fibras possuem aproximadamente $90 \%$ de proteína de queratina. Essa estrutura é formada de alfa-aminoácidos, que são constituídos de uma estrutura helicoidal de materiais de alta resistência. Tem como estrutura um carbono central (carbono alfa) ligado a: grupos funcionais amina $\left(\mathrm{NH}_{2}\right)$ e ácido carboxílico $(\mathrm{COOH})$; átomos de hidrogênio e alguns aminoácidos podem conter enxofre em sua estrutura (Moore et al., 2005; Campbell, 2006), Figura 2. Estrutura dos aminoácidos. 
<smiles>[R]C(N)C(=O)O</smiles>

Figura 2 - Forma geral dos aminoácidos. (FRANCISCO JUNIOR; FRANCISCO 2006, adaptado)

As penas são biodegradáveis, possuem nós e ganchos, são impermeáveis a água e são ocas, possibilitando o isolamento térmico (Wool \& Sun, 2005), Figura 3. Estas características podem melhorar as propriedades estruturais e aumentar a aderência com resinas na fabricação de compósitos. Um compósito a ser fabricado, será leve e novos materiais, dependendo da proporção da KF e o tipo de resina, a sua densidade pode ser alterado de acordo com a aplicação final do produto.

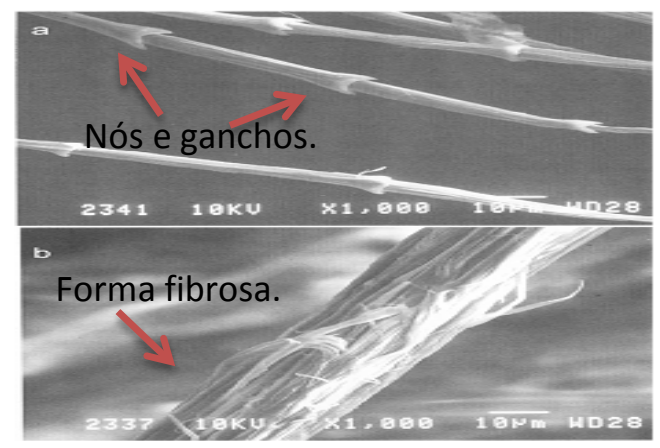

Figura 3 - Microscopias das penas: (a) Fibra de Queratina e (b) um pequeno eixo oco. FONTE: Wool \& Sun (2005)

Devido os aminoácidos, serem heterogêneos, pode influenciar as propriedades mecânicas do produto final, sendo necessário sintetiza-las, restando somente átomos de carbono assim formando um precursor na fabricação de fibra de carbono, necessitando apenas do tratamento químico ou térmico adequado.

De acordo com Hadjizadeh; Ajji; Bureau, 2010, o tratamento das fibras com Hidróxido de Sódio $(\mathrm{NaOH})$ é um método simples e barato para alterar as suas propriedades superficiais. Resultando no aumento da porosidade, diminuição da espessura e densidade. Entretanto esse tratamento precisa ser realizado em curto tempo, baixa concentração e temperatura, pois o excesso de poros nas superfícies das fibras resulta na diminuição da resistência a tração bem como o modulo de elasticidade.

Novas propriedades são evoluídas de materiais carbonizados, como as fibras de carbono, materiais fibrosos de carbono em nanoescalas e a grafita, capazes de suportar altas temperaturas sem perder suas propriedades físico-químicas e mecânicas. As fibras de Poliacrilonitrila (PAN), Rayon (viscose), são usadas como precursores na fabricação de fibras de carbono em grande escala. Desta forma a fibra de carbono provém da pirólise de materiais carbonáceos. 0 diferencial em carbonizar as penas será o aumento em relação à força-peso, podendo permitir compósitos mais fortes, mais leves e ecologicamente corretos.

As análises térmicas são controladas em função da temperatura e ou tempo, enquanto a temperatura da amostra é submetida a uma programação controlada (PICCOLI et al., 2006). 
Sendo assim uma análise apropriada para se descobrir um melhor ciclo de carbonização e a avaliação do comportamento do ciclo térmico imposto à fibra de penas torna-se uma ferramenta para obter fibras carbonizadas de qualidade.

A carbonização é um processo que reduz o material orgânico a carbono, e pode ser feito por pirólise. O material é exposto à alta temperatura, por longos períodos de tempo, para fornecer ao mesmo a energia de ativação necessária para quebrar as ligações não carbono-carbono, em um ambiente com pouco ou nenhum oxigênio. Esse método reduzirá a oxidação e preservará a integridade da estrutura geral do material, enquanto a sua massa é reduzida e, além disso, propriedades novas evoluíram a partir de material carbonizado. Porém o processo de carbonização torna-se complicado. Segundo Wool \& Sun, 2005 a integridade das ligações carbono-nitrogênio deve ser preservada para manter a estrutura fibrosa do precursor. Portanto são necessários diversos parâmetros a partir de análise termogravimétrica na concepção dos ciclos.

\section{MATERIAIS E MÉTODOS}

\section{MATERIAIS}

As penas de frangos (KF), utilizadas nesse trabalho, são oriundas de indústria avícola local. As demais amostras foram desenvolvidas a partir delas por tratamentos químico $(\mathrm{NaOH})$ e térmicos (SCFC, SCFD e CF).

\section{MÉTODOS}

\section{PRÉ TRATAMENTO DA KF}

A KF foi lavada com água corrente, retirada suas impurezas, tratadas com detergente neutro e novamente lavadas com água corrente. Em seguida foram secadas a $100^{\circ} \mathrm{C}$ por 2 horas, numa estufa TE39411 da Tecnal com circulação de ar. Após secagem uma parte da KF foi armazenada para ser tratada, quimicamente e termicamente. A outra parte foi triturada, Figura 4, e armazenada para ser analisada por curvas de TG e DTG.

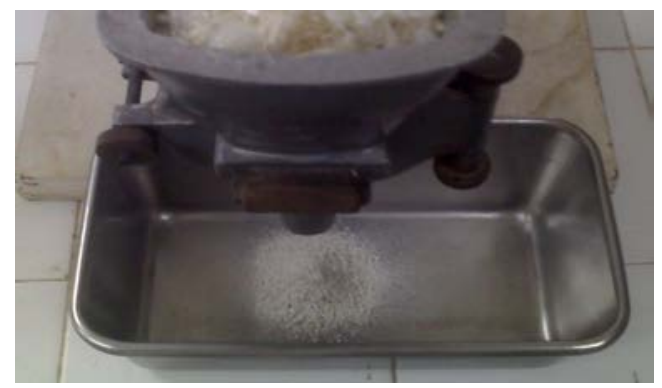

Figura 4 - KF no triturador de marca Culatto.

\section{TRATAMENTO QUÍMICO DA KF}

As amostras da $\mathrm{KF}$ foram tratadas com uma solução aquosa de $2 \%(\mathrm{NaOH})$ a temperatura de $100^{\circ} \mathrm{C}$ num autoclave vertical, PHOENIX, por uma hora, Figura 4.a. Em seguida as KF, tratada 
quimicamente $(\mathrm{KFNaOH})$, foram lavadas na água corrente até a neutralização do pH, Figura 5. b. Para garantir que todo $\mathrm{NaOH}$ tivesse sido eliminado das fibras, verificou o $\mathrm{pH}$ inicial da água, antes do tratamento, utilizando papel de tornassol e lavou-se o resíduo até que o $\mathrm{pH}$ da água residual votasse a ser $\mathrm{o} \mathrm{pH}$ inicial. Após essa etapa, as $\mathrm{KFNaOH}$ foram secadas, trituradas igualmente a KF e armazenadas para serem analisadas por TGA/DTG.

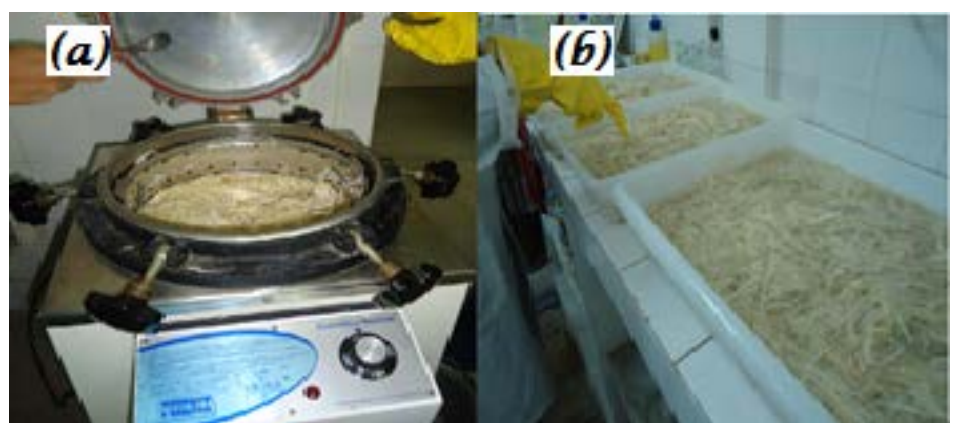

Figura 5 - (a) KF em autoclave com solução de $\mathrm{NaOH}$ a $2 \%$ e a (b) sendo lavadas após tratamento.

\section{SEMICARBONIZAÇÃO DA KF}

Inicialmente pesaram-se as KF e submeteu-as em estufa TE39411 da Tecnal, com circulação de ar atmosférico, por $24 \mathrm{~h}$ a $220^{\circ} \mathrm{C}$, sem respeitar taxas de aquecimento e resfriamento. Optou-se em distribuir o material na parte superior e inferior da estufa, Figura 6. Após esse período as KF semicarbonizadas, Figura 7, foram pesadas, trituradas e armazenadas da mesma forma e com o mesmo objetivo das $\mathrm{KF}$ e $\mathrm{KFNaOH}$.

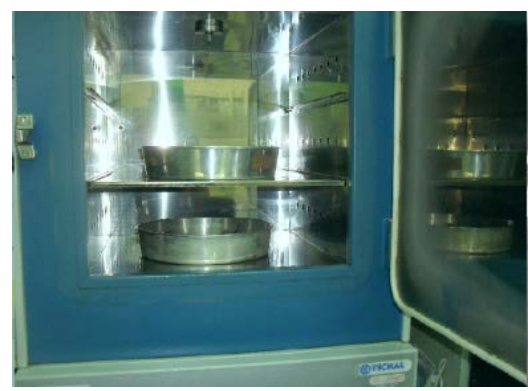

Figura 6 - Estufa com circulação de ar atmosférico.

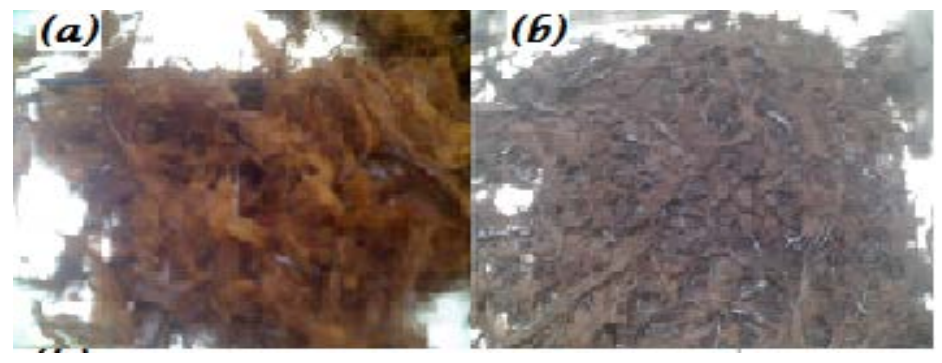

Figura 7 - KF semicarbonizadas; mais claras -(a) SCFC e mais escuras - (b) SCFD.

4. ANÁLISE TÉRMICA DA KF, KFNaOH, SCFC E SCFD

As análises por TG foram conduzidas com o intuito de identificar o limite de temperatura 
até a qual se observa a degradação das fibras, como também a oxidação das mesmas, em um equilíbrio da taxa da degradação com o aumento de temperatura necessária para projetar um ciclo de carbonização que obtenha maior quantidade de resíduo com ligações carbono-carbono, porém que preserve as ligações carbono-nitrogênio para assim manter a KF mais resistente e manter sua estrutura fibrosa. Essa etapa foi realizada no Laboratório de Análise Térmica e Eletroanalítica (LATEL) da UFRN. O equipamento utilizado foi tga50 da Shimadzu capaz de operar da temperatura ambiente até $900^{\circ} \mathrm{C}$ com razão de aquecimento de $10^{\circ} \mathrm{C} \mathrm{mm}^{-1}$, com taxa de aquecimento de $10^{\circ} \mathrm{Cmin}^{-1}$, sob atmosfera de ar sintético e nitrogênio com fluxo de $20 \mathrm{mlmin}^{-1}$. Utilizou-se para cada amostra, entre 10 e $0,5 \mathrm{mg}$, Figura 8. Foram obtidas às curvas de TG e DTG, que auxilia no estudo das temperaturas máximas da perda de massa.

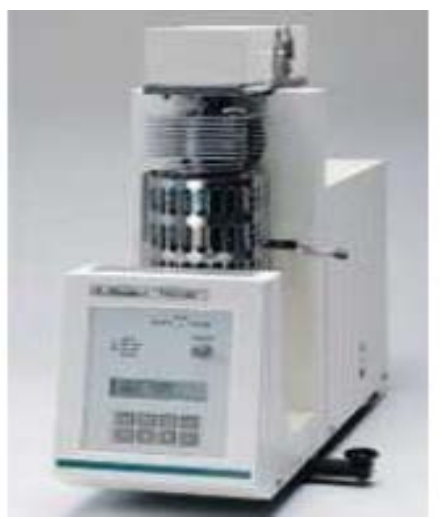

Figura 8 - Equipamento da análise termogravimétrica realizada nesse estudo.

\section{PIRÓLISE}

Entretanto, o ciclo de carbonização por pirólise, foi obtido a partir da observação física das amostras e de suas curvas de TG/DTG. A KF foi pesada e submetida ao ciclo de carbonização em forno resistivo tubular MAITEC FTE-1600/H, sob atmosfera de argônio com fluxo de $100 \mathrm{~cm}^{3} \mathrm{~mm}^{-1}$ no Laboratório de propriedades físicas de materiais cerâmicos da UFRN, Figura 9.

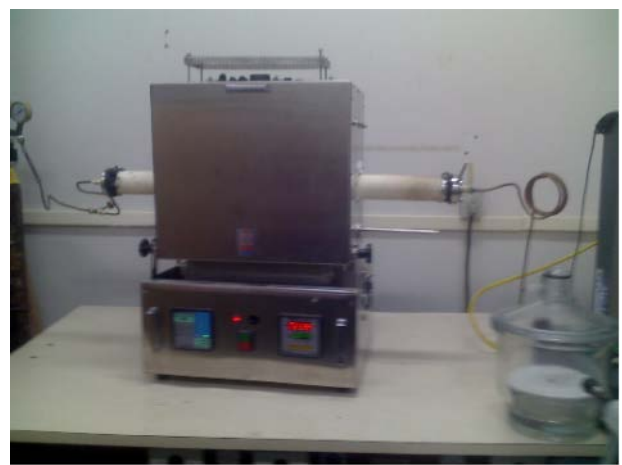

Figura 9 - Forno tubular.

A KF foi submetida ao patamar de $220^{\circ} \mathrm{C}$ por 24 horas. Em seguida a temperatura foi elevada a $450^{\circ} \mathrm{C}$ por mais 1 hora, respeitando as taxas de aquecimento e resfriamento de $3^{\circ} \mathrm{C} \cdot \mathrm{min}^{-1}$, Figura 10. Ver Figura 11, a KF e CF. 


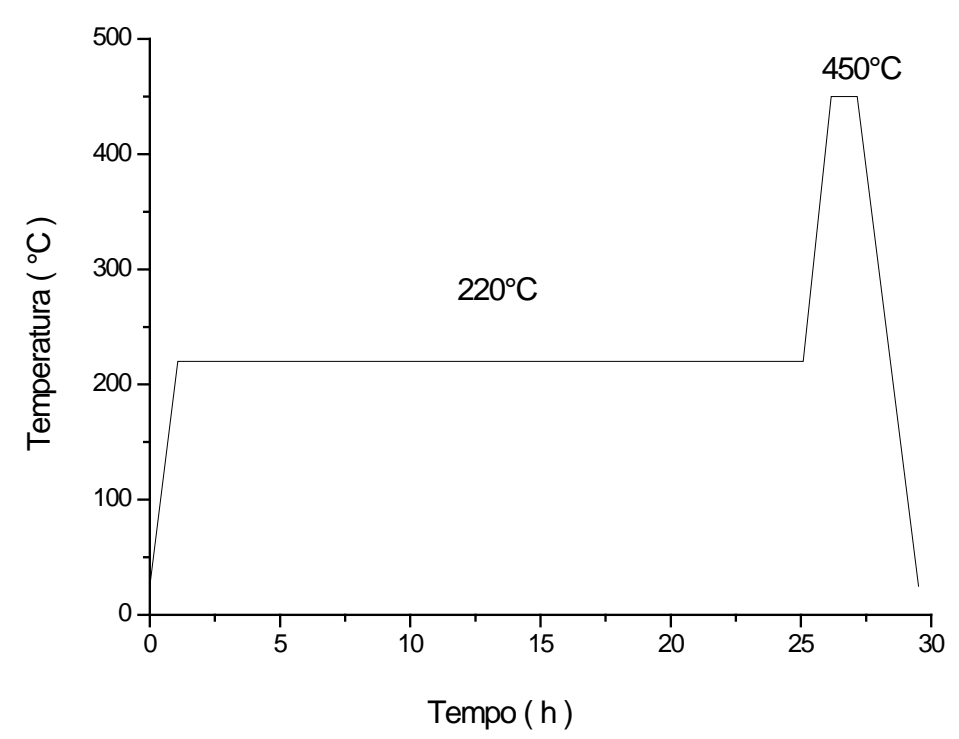

Figura 10 - Evolução da temperatura em função do tempo do ciclo de pirólise da KF.

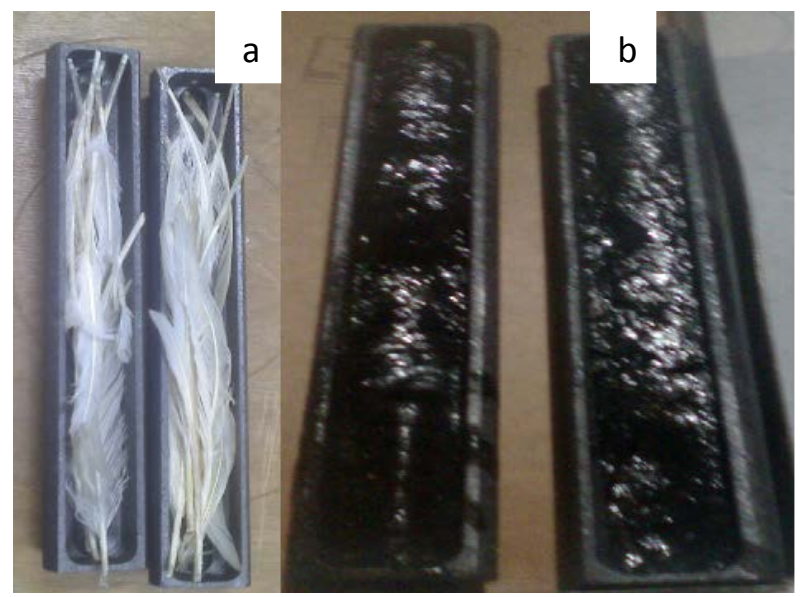

Figura 11 - (a) KF e (b) CF.

Após esse processo a CF foi pesada e armazenada para análise de TG/DTG, conforme descrito no item anterior, para ser comparada com as demais fibras, e assim determinar o aumento ou a diminuição da estabilidade térmica da mesma.

\section{RESULTADOS E DISCUSSÕES}

\section{RESULTADOS PARA OBTENÇÃO DO CICLO DE PIRÓLISE}

A perda de massa após trituração da $\mathrm{KF}, \mathrm{KFNaOH}$ foi de $27 \%, 30 \%$, respectivamente. Entretanto a $\mathrm{KFNaOH}$ não apresentou diferença significativa de perda de massa em relação a KF, também percebido após análise de TGA, Figura 12 (b) e (d), com rendimentos totais de aproximadamente de $22 \%$ e $24 \%$, respectivamente.

Já as SCFC e SCFD apresentaram estruturas semelhantes às originais, porém apresentaram estruturas quebradiças, e respectivamente, colorações entre marrom claro e escuro. A perda de massa após esse processo foi de $30 \%$ e $40 \%$, respectivamente. Pelas curvas de TG/DTG, verifica- 
se a diferença entre as resistências térmicas com rendimentos totais de aproximadamente de $29 \%$ e 18\%, respectivamente, Figura 12 (f), (h). A SCFD ficou com resistência térmica menor, isso é devido a maior oxidação do material. Sendo assim o processo utilizado em sua obtenção não é suficiente para sintetizar o material esperado (material que apesar de preservar a estrutura da $\mathrm{KF}$, seja mais resistente e mais homogêneo). Lembrando que a influência do ar na estrutura da KF nesse processo de oxidação deu inicio a partir de $220^{\circ} \mathrm{C}$, sendo comprovadas pela comparação da Figura 12 (a), (c), (e) e (g) com a Figura 12 (b), (d), (f) e (h). Comparando as curvas de TG/TDG, Figura 12 (a)(c),(e) e (g) pode-se visualizar uma melhor estabilidade térmica da SCFC com a KF e $\mathrm{KFNaOH}$, no entanto nem sempre isso vai ser garantido, pois o processo foi realizado sem tempo, temperatura e atmosfera controlada.

Á resistência a temperatura é consideravelmente maior, quando as amostras são analisadas sob atmosfera controlada, sendo melhor visualizada a partir de $400^{\circ} \mathrm{C}$ Figura 12 (a) e (b). Observou-se que a KF perde cerca de $6 \%$ de sua massa inicial até $100^{\circ} \mathrm{C}$, e isso é devido à evaporação a água. Como a temperatura continua a subir, começa a degradação do material em aproximadamente $220^{\circ} \mathrm{C}$, representando uma perda em torno de $3 \%$ a mais que do resíduo a 100 ${ }^{\circ} \mathrm{C}$. Entre $220^{\circ} \mathrm{C}$ e $450^{\circ} \mathrm{C}$, verifica-se um acentuado declive, relacionado à degradação do material em $56 \%$ a mais que do resíduo entre temperatura ambiente e $220^{\circ} \mathrm{C}$. A $220^{\circ} \mathrm{C}$, pode-se perceber um ponto de equilíbrio de carbonização que produz um resíduo de fração menos que os valores expressos pelo rastreamento de toda análise. A $450^{\circ} \mathrm{C}$ obtém-se uma perda em torno de $66 \%$ da massa original. Já em $550^{\circ} \mathrm{C}$ à perda de espécies químicas é bem mais significante chegando a $86 \%$, Figura 12 (b). Comparando o rendimento total da massa até $900^{\circ} \mathrm{C}$, da $\mathrm{KF}$ em atmosferas de Ar sintético e $\mathrm{N}_{2}$, temos $5 \%$ e $22 \%$, respectivamente, Figura 12 (a) e (b).
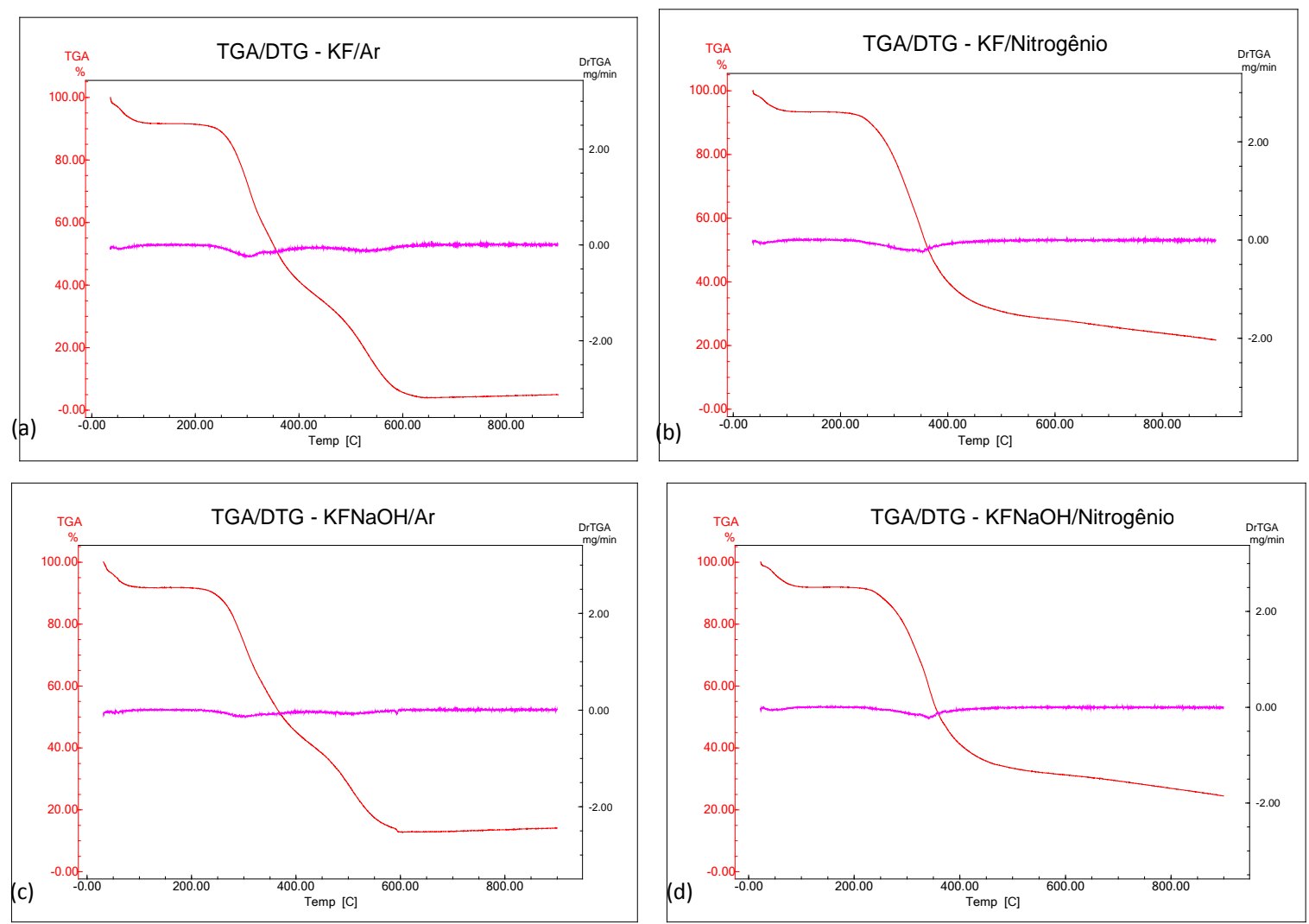

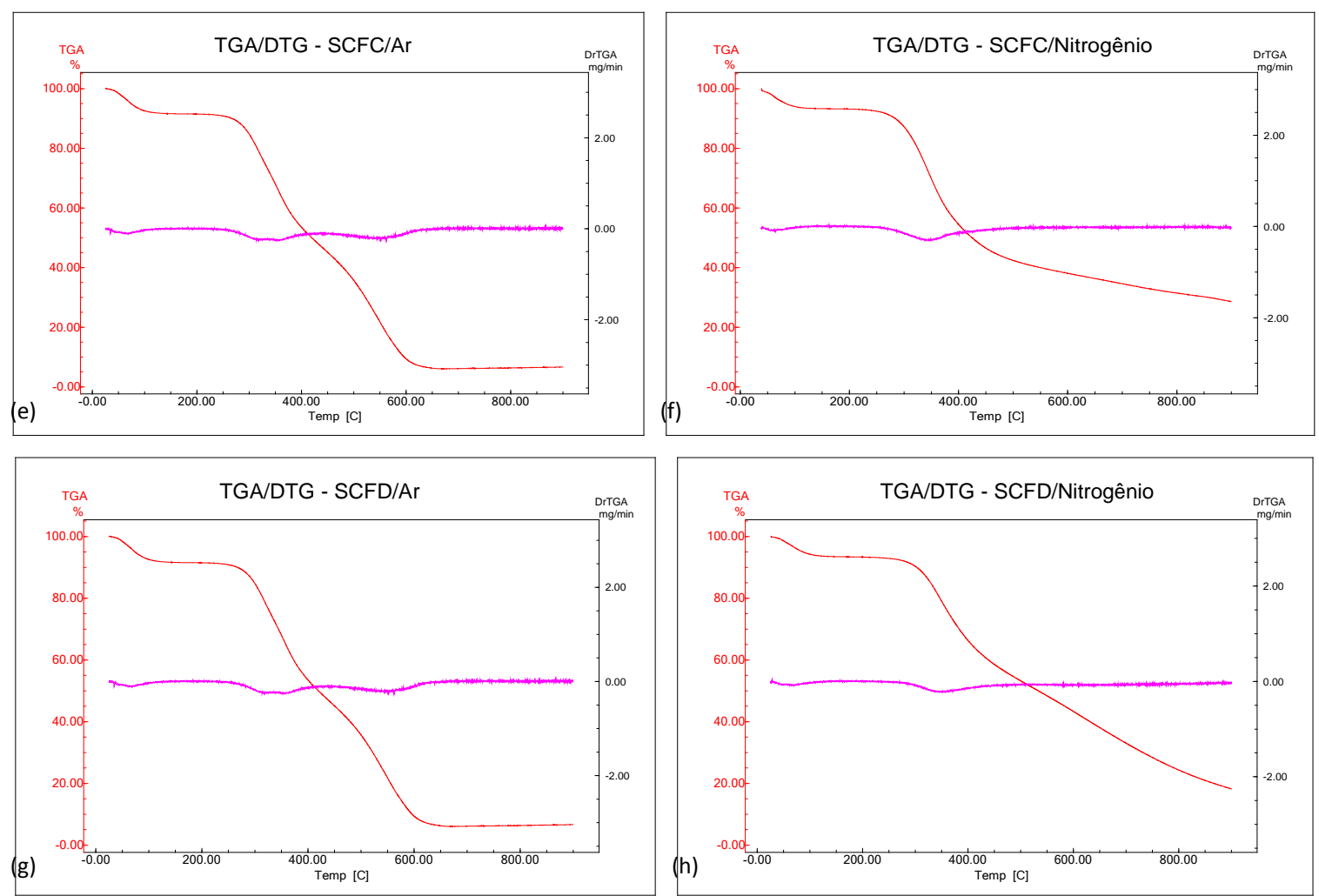

Figura 12 - Curvas obtidas por TGA (vermelho) e sua derivada, DTG (rosa) para as amostras analisadas nesse item, em Ar sintético / Nitrogênio.

Com base nessas informações optou-se pela KF como material precursor na obtenção de material carbonoso de melhor qualidade, pelo processo em atmosfera controlada e respeitando taxas de aquecimento e resfriamento, como também o acréscimo na temperatura para $450^{\circ} \mathrm{C}$ para assim aumentar o teor de ligações carbono-carbono e tentar preservar ligações carbononitrogênio. $\mathrm{O}$ tempo de 24 horas a $220^{\circ} \mathrm{C}$ foi escolhido com a intenção de obter um processo gradativo.

\section{RESULTADO DA ANÁLISE TÉRMICA DA CF}

Após o processo de pirólise, a CF apresentou aspectos: negro, brilhoso, o que não foi percebido nas outras amostras e obteve $36 \%$ da massa original (KF). Foi um menor rendimento comparado com todas as outras amostras, porém ao observar as curvas TG/DTG, ela apresentou maior estabilidade térmica. O seu resíduo final foi em torno de $30 \%$, Figura 13 (b), maior que as $\mathrm{KF}(22 \%), \mathrm{KFNaOH}(24 \%), \mathrm{SCFC}(29 \%)$ E SCFD (19\%). Como em todas as outras amostras, as análises realizadas para $\mathrm{CF}$, sob fluxo de $\mathrm{N}_{2}$, limitaram a oxidação do material, contribuindo para o aumento na resistência térmica das fibras. O rendimento total da massa Ar sintético foi de $4 \%$, Figura 13 (a). Porém essa diferença na resistência térmica da $C F$, só é perceptível em torno de $450^{\circ} \mathrm{C}$. 

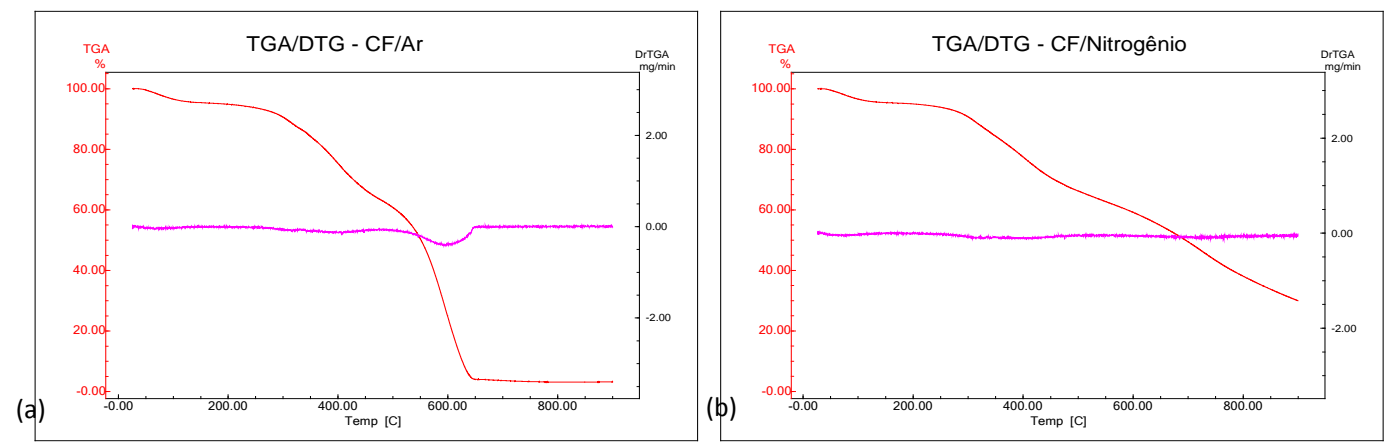

Figura 13 - Curvas obtidas por TGA (vermelho) e sua derivada, DTG (rosa) para a CF em (a) Ar sintético / (b) Nitrogênio.

\section{CONCLUSÕES}

Os tratamentos dados a KF e as análises térmicas (TG/DTG) evidenciaram que a pirólise foi um melhor processo para determinação de materiais carbonosos de melhor qualidade. Todas as amostras preparadas e analisadas nesse estudo podem ser utilizadas como reforços, em materiais compósitos para fins de utilização a uma temperatura até $450^{\circ} \mathrm{C}$. Acima dessa temperatura os compósitos poderão oxida-se em ar atmosférico e consequentemente degradará facilmente. Entretanto a CF dará maior resistência térmica aos materiais compósitos. Diante do objetivo desse trabalho foi possível desenvolver um material inédito, com destino ecologicamente correto e de grande valor agregado.

\section{AGRADECIMENTOS}

Os autores do trabalho agradecem ao Laboratório de Propriedades Físicas de Materiais Cerâmicos, pelo processo de pirólise da KF. E ao Laboratório de Análise Térmica e Eletroanalítica (LATEL), pelas análises térmicas realizadas nesse estudo. Ambos da Universidade Federal do Rio Grande do Norte (UFRN).

\section{REFERÊNCIAS BIBLIOGRÁFICAS}

1. AVEWORLD. Brasil, o maior exportador de frangos do mundo sediará o XXIV Congresso Mundial de Avicultura. Disponível em: <http://www.aveworld.com.br/noticias/post/brasil-o-maiorexportador-de-frangos-do-mundo-sediara-o-xxiv-congresso-mundial-de-vicultura>. Acesso em: 01 abr. 2012.

2. CAMPBELL, M. K. Bioquímica. 3o edição, Artmed, 2006.

3. FRANCISCO JUNIOR, W. E.; FRANCISCO, W., Proteína: Hidrólise, Precipitação e um Tema para o Ensino de Química. Química Nova Na Escola, [S. I.], n. , p.12-16, 09 out. 2006. Disponível em: <http://qnesc.sbq.org.br/online/qnesc24/ccd1.pdf> Acesso em: 15 jun 2012.

4. HADJIZADEH, A.; AJJI, A.; BUREAU, M. N. Preparation and characterization of $\mathrm{NaOH}$ treated micro-fibrous polyethylene terephthalate nonwovens for biomedical application. Journal of The Mechanical Behavior Of Biomedical Materials, [s. L.], p. 574-583. 9 July 2010. Disponível em: <http://www.sciencedirect.com/science/article/pii/S1751616110000871>. Acesso em: $14 \mathrm{abr}$. 2012. 
5. IBGE - Instituto Brasileiro De Geografia e Estatística (Brasil). Abates de frangos e suínos e produção de leite e ovos crescem em 2011. Disponível em: $<$ http://www.ibge.gov.br/home/presidencia/noticias/noticia visualiza.php?id noticia=2107\&id pagin $\mathrm{a}=1>$. Acesso em: 15 jun.2012a.

6. IBGE - Instituto Brasileiro De Geografia e Estatística (Brasil ). Produção Animal: $4^{\circ}$ Trimestre de 2011 e Acumulado do Ano. Disponível em: < http://www.ibge.gov.br/home/presidencia/noticias/imprensa/ppts/00000008050103112012013329 746408.pdf>. Acesso em: 15 jun. 2012b.

7. MOORE, G. R. P., et al,. Queratina de Penas de frango: extração, caracterização e obtenção de filmes", Vol. 26, no.2, Campinas, Brasil, 2005. Disponível em: <http://www.scielo.br/pdf/cta/v26n2/30192.pdf >. Acesso em: 23/04/2012.

8. PICCOLI, R., et al. Características das principais técnicas analíticas aplicadas à caracterização de materiais. In: congresso brasileiro de engenharia e ciência dos materiais, 17., 2006, Foz do Iguaçu, Pr, Brasil. Caracterização de materiais. Foz do Iguaçu: Metallum, 2006. Vol. 102, p. 028 -028. Disponível em: <http://www.metallum.com.br/17cbecimat/resumos/17cbecimat-102028.pdf>. Acesso em: 28 ago. 2011.

9. WOOL, R. P., SUN, X. S., 2005, Bio-Based Polymers and Composites -ISBN: 0127639527 Publisher: Elsevier Science \& Books- Pub. Date: July 2005. 